\title{
Спектроскопия одиночных AllnAs- и (111)InGaAs-квантовых точек
}

\author{
(C) И.А. Деребезов ${ }^{1,2}$, В.А. Гайслер ${ }^{1}$, А.В. Гайслер ${ }^{1}$, Д.В. Дмитриев ${ }^{1}$, А.И. Торопов ${ }^{1}$, \\ M. von Helversen ${ }^{3}$, C. de la Haye ${ }^{3}$, S. Bounouar ${ }^{3}$, S. Reitzenstein ${ }^{3}$ \\ ${ }^{1}$ Институт ффизики полупроводников им. А.В. Ржанова \\ Сибирского отделения Российской академии наук, \\ 630090 Новосибирск, Россия \\ ${ }^{2}$ Сибирский государственный университет телекоммуникаций и информатики, \\ 630102 Новосибирск, Россия \\ ${ }^{3}$ Technische Universität Berlin, Institut für Festkörperphysik Eugene-Wigner-Gebäude, \\ D-10623 Berlin, Federal Republic of Germany \\ E-mail: derebezov@isp.nsc.ru
}

(Получена 25 апреля 2018 г. Принята к печати 7 мая 2018 г.)

Исследована система квантовых точек на основе AlInAs и (111)InGaAs. Использование широкозонных твердых растворов $\mathrm{Al}_{x} \operatorname{In}_{1-x} \mathrm{As}$ в качестве основы квантовых точек позволило существенно расширить спектральный диапазон излучения в коротковолновую область, включая участок длин волн вблизи 770 нм, представляющий интерес для разработки аэрокосмических систем квантовой криптографии. Исследована тонкая структура экситонных состояний AlInAs- и (111)InGaAs-квантовых точек. Показано, что для набора квантовых точек расщепление экситонных состояний сравнимо с естественной шириной экситонных линий, что представляет интерес для разработки излучателей фотонных пар на их основе.

DOI: 10.21883/FTP.2018.11.46593.15

\section{1. Введение}

Неклассические излучатели света являются важнейшим ресурсом квантовых информационных систем [1]. Структуры на основе полупроводниковых квантовых точек (КТ) представляют большой интерес для практической реализации таких устройств [2,3]. Одиночная КТ может быть использована в качестве основы излучателя одиночных фотонов (ИОФ). Одиночная квантовая точка может быть и источником фотонных пар (ИФП), запутанных по поляризации, в процессе каскадной рекомбинации биэкситона и экситона в случае, если экситонные состояния вырождены по энергии или же их расщепление не превышает естественной ширины экситонных уровней $\Gamma$ [4-6]. В КТ, синтезированных на подложках (001)GaAs, расщепление экситонных состояний, как правило, многократно превышает естественную ширину экситонных уровней $[7,8]$. В отличие от КТ, синтезированных на (001)GaAs-подложках, пьезоэлектрический потенциал для КТ, выращенных на (111)GaAs-подложках направлен вдоль направления роста [9] и не понижает симметрию ниже $C_{3 v}$ вдоль основания КТ. В этом случае величина сверхтонкого расщепления будет нулевой. Поскольку КТ, выращенные на подложке (111) GaAs, обладают $C_{3 v}[9,10]$ симметрией, то такие КТ могут быть источником пар запутанных фотонов $[11,12]$. К настоящему моменту времени наиболее изученной является система InAs-квантовых точек, уникальной особенностью которой является широкий спектральный диапазон, включающий в себя первый и второй телекоммуникационные стандарты. Расширение спектрального диапазона излучения квантовых точек в коротковолновую область представляет интерес для создания источников излучения систем атмосферной или аэрокосмической кванто- вой криптографии. Оптимальным участком длин волн для данных систем признан участок вблизи 770 нм [13].

В данной работе представлены результаты исследования тонкой структуры экситонных состояний квантовых точек на основе $\mathrm{Al}_{x} \operatorname{In}_{1-x} \mathrm{As}_{2} / \mathrm{Al}_{y} \mathrm{Ga}_{1-y} \mathrm{As}$ и квантовых точек (111)InGaAs.

\section{2. Описание структур и методик исследования}

Структуры на основе $\mathrm{Al}_{x} \mathrm{In}_{1-x} \mathrm{As} / \mathrm{Al}_{y} \mathrm{Ga}_{1-y} \mathrm{As}$ выращивались на установке методом молекулярно-лучевой эпитаксии (МЛЭ) на подложках (001)GaAs. На поверхности подложки выращивался буферный $\mathrm{GaAs}$ слой толщиной 0.3 мкм, после чего производился синтез слоев образующих исследованную структуру. Структура содержала два 40-нанометровых слоя $\mathrm{Al}_{0.7} \mathrm{Ga}_{0.3} \mathrm{As}$, препятствующих диффузии фотовозбужденных носителей заряда, и 200-нанометровый слой $\mathrm{Al}_{y} \mathrm{Ga}_{1-y} \mathrm{As}$, находящийся между ними. В середине $200 \mathrm{Hм} \mathrm{Al}_{y} \mathrm{Ga}_{1-y}$ As-слоя, поглощающего большую часть мощности возбуждающего лазера, находился слой $\mathrm{Al}_{x} \mathrm{In}_{1-x} \mathrm{As} \mathrm{KT.} \mathrm{Были} \mathrm{изучены}$ $\mathrm{Al}_{x} \mathrm{In}_{1-x}$ As KT с составом в интервале $x=0-0.3$ с шагом 0.05. При этом состав слоев $\mathrm{Al}_{y} \mathrm{Ga}_{1-y} \mathrm{As}$ задавался согласно $y \approx 1.7 x$. Слой $\mathrm{Al}_{x} \mathrm{In}_{1-x} \mathrm{As}$ КТ выращивался по механизму Странского-Крастанова при температуре $T=505^{\circ} \mathrm{C}$. На поверхности $\mathrm{Al}_{y} \mathrm{Ga}_{1-y} \mathrm{As}$ синтезировался слой $\mathrm{Al}_{x} \operatorname{In}_{1-x} \mathrm{As}$ критической толщины ( 2 монослоя $(\mathrm{MC}))$, по достижении которой начинал формироваться массив самоорганизованных $\mathrm{Al}_{x} \mathrm{In}_{1-x} \mathrm{As}$ KT. Скорость роста $\mathrm{Al}_{x} \mathrm{In}_{1-x} \mathrm{As}$-слоев составляла $0.045 \mathrm{MC} / \mathrm{c}$. Переход от двумерного механизма роста к трехмерному контролировался методом дифракции быстрых электронов. При достижении критической толщины процесс роста 
$\mathrm{Al}_{x} \mathrm{In}_{1-x} \mathrm{As}$ прекращался, и в течение времени $\tau_{\mathrm{GI}}$ формировался массив $\mathrm{Al}_{x} \operatorname{In}_{1-x}$ As KT по механизму Оствальда [14-18], после чего слой $\mathrm{Al}_{x} \mathrm{In}_{1-x} \mathrm{As}$ КТ заращивался $\mathrm{Al}_{y} \mathrm{Ga}_{1-y}$ As. Время ростовой паузы $\tau_{\mathrm{GI}}=10 \mathrm{c}$.

Структуры, содержащие (111)InGaAs KT, были синтезированы методом МЛЭ на $2^{\circ}$ отклоненных подложках $n^{+}$-GaAs с ориентацией (111). На поверхности подложки выращивался буферный GaAs слой толщиной 0.3 мкм. Структура содержала два 50-нанометровых слоя $\mathrm{Al}_{0.6} \mathrm{Ga}_{0.4} \mathrm{As}$, препятствующих диффузии фотовозбужденных носителей заряда, и 160-нанометровый слой GaAs, находящийся между ними. В середине 160нанометрового GaAs слоя находился слой содержащий (111)InGaAs КТ. Слой (111)InGaAs КТ синтезировался по механизму „капельной“ эпитаксии [19,20], на поверхность GaAs осаждался In без потока As, после чего в потоке As производилась кристаллизация капель In для формирования (111)InGaAs KT. С целью определения влияния условий роста на величину расщепления экситонных состояний (111)InGaAs КТ была синтезирована серия образцов. При синтезе (111)InGaAs КТ варьировались температура подложки $T_{\text {sub }}$ в интервале от 460 до $500^{\circ} \mathrm{C}$, время осаждения In $t_{Q D}$ в интервале от 30 до 36 с (что обеспечивало толщину In слоя $h_{\text {In }}$ от $\sim 2.0$ до $\sim 2.4 \mathrm{MC}$ ) и время ростовой паузы $\tau_{\mathrm{GI}}$ в интервале от 0 до $60 \mathrm{c}$.

Структуры, содержащие КТ, исследовались методами макро- и микрофотолюминесценции, площадь пятна возбуждающего лазера на поверхности структуры составляла 3000 и 3 мкм ${ }^{2}$ соответственно. Для возбуждения люминесценции использовалось излучение Nd:YAG-лазера с длиной волны 532 нм, работающего в непрерывном режиме. Люминесценция регистрировалась с помощью однократного монохроматора, оснащенного охлаждаемым Ge-pin фотодиодом (макролюминесценция), или с использованием тройного монохроматора „TriVista-555“ с охлаждаемой ПЗС (прибор с зарядовой связью) матрицей $\mathrm{Si}$-фотоприемников (микролюминесценция). Интерпретация пиков люминесценции одиночных КТ проводилась с использованием зависимостей интенсивностей пиков от мощности излучения возбуждающего лазера. При наименьших мощностях возбуждения в спектрах люминесценции в первую очередь проявляются пики экситонов $X$ и зависимость их интенсивности от мощности линейна. При бо́льших мощностях в спектрах появляются пики биэкситонов $X X$, интенсивность которых возрастает по квадратичному закону от плотности мощности лазера [20,21]. В идеальных КТ оптически активные экситоны, образованные основными электронными и дырочными состояниями с моментами $(+1 / 2 ;-3 / 2)$ и $(-1 / 2 ;+3 / 2)$, вырождены по энергии. В реальных КТ это вырождение снимается за счет отклонения КТ от идеальной формы, а также влияния пьезопотенциала, индуцированного встроенными механическими напряжениями [20,21]. Величина расщепления экситонных уровней $\Delta E_{F S}$ определяется набором факторов, таких, как степень отклонения формы КТ от идеальной и влияние пьезопотенциала, значение которого возрастает с увеличением размера КТ [21]. При $\Delta E_{F S}$, значительно превосходящих $\Gamma_{X}$, излучение экситона и биэкситона линейно поляризовано вдоль кристаллографических направлений [110] и [1ㅣ], в случае если КТ синтезирована на подложке с ориентацией (001). Соответственно спектры люминесценции одиночной (001) КТ, записанные для поляризаций вдоль направлений [110] и [1ํㅣ, будут содержать пики экситонов $X[110]$ и $X[1 \overline{1} 0]$ и биэкситонов $X X[110]$ и $X X[1 \overline{10}]$, смещенных друг относительно друга, на величину $\Delta E_{F S}$, что позволяет непосредственно из спектров излучения определять параметр расщепления экситонных состояний $\Delta E_{F S}$. В данной работе величина $\Delta E_{F S}$ определялась как разница энергий фотонов $X[110]$ и $X[1 \overline{1} 0]$, отвечающих экситонной рекомбинации в $\mathrm{Al}_{x} \operatorname{In}_{1-x} \mathrm{As}$ KT. В структурах, содержащих (111) In $(\mathrm{Ga}) \mathrm{As}$ KT, величина $\Delta E_{F S}$ определялась из поляризационных измерений, в которых использовались фиксированный линейный поляризатор, расположенный непосредственно перед входной щелью монохроматора, и $\lambda / 2$ вращающаяся пластина для вращения плоскости поляризации.

Идеальные КТ с вырожденными по энергии экситонными состояниями $\left(\Delta E_{F S}=0\right)$ и КТ с малым расщеплением экситонных состояний $\left(\Delta E_{F S} \approx \Gamma=\hbar / \tau_{X}\right)$ могут быть источниками пар, запутанных по поляризации фотонов [21], в процессе каскадной рекомбинации биэкситона и экситона. Согласно литературным данным, время жизни экситона $\tau_{X}$ при криогенных температурах составляет $\sim 10^{-9}$ с $[20,21]$, что задает ширину экситонного пика $\tau_{X}$ на уровне $10^{-6}{ }_{\ni}$. Таким образом, для разработки источника пар, запутанных по поляризации фотонов, представляют интерес КТ, в которых величина $\Delta E_{F S}$ не превышает нескольких мкэВ. Для поиска КТ, отвечающих данному требованию, погрешность определения $\Delta E_{F S}$ должна быть также на уровне единиц мкэВ, в данной работе погрешность в определении параметpa $\Delta E_{F S}$ составляла $\sigma_{F S}= \pm 1.6$ мкэВ, что сравнимо с естественной шириной экситонных пиков $\Gamma_{X}$.

Статистика излучения одиночных КТ изучалась с использованием интерферометра Хэнбери Брауна-Твисса (ХБТ), который был собран по классической схеме [20,21]. Излучение КТ, проходя первый монохроматор „TriVista-555“, направлялось на светоделительную неполяризующую призму „50/50 CCM1-BS014““, которая разделяла падающий световой поток строго поровну. Излучение в каждом из каналов регистрировалось счетчиками фотонов на основе $\mathrm{Si}$-лавинных фотодиодов „PerkinElmer SPCM-AQRH-15“, временны́е характеристики излучения анализировались с использованием счетчика совпадений „PicoHarp 300“.

\section{3. Экспериментальные результаты}

Спектры макрофотолюминесценции структур, содержащих $\mathrm{Al}_{x} \mathrm{In}_{1-x} \mathrm{As}_{2} / \mathrm{Al}_{y} \mathrm{Ga}_{1-y} \mathrm{As}$ KT различного состава при $T=295 \mathrm{~K}$, представлены на рис. $1, a$. Представленные данные демонстрируют расширение спектрального 

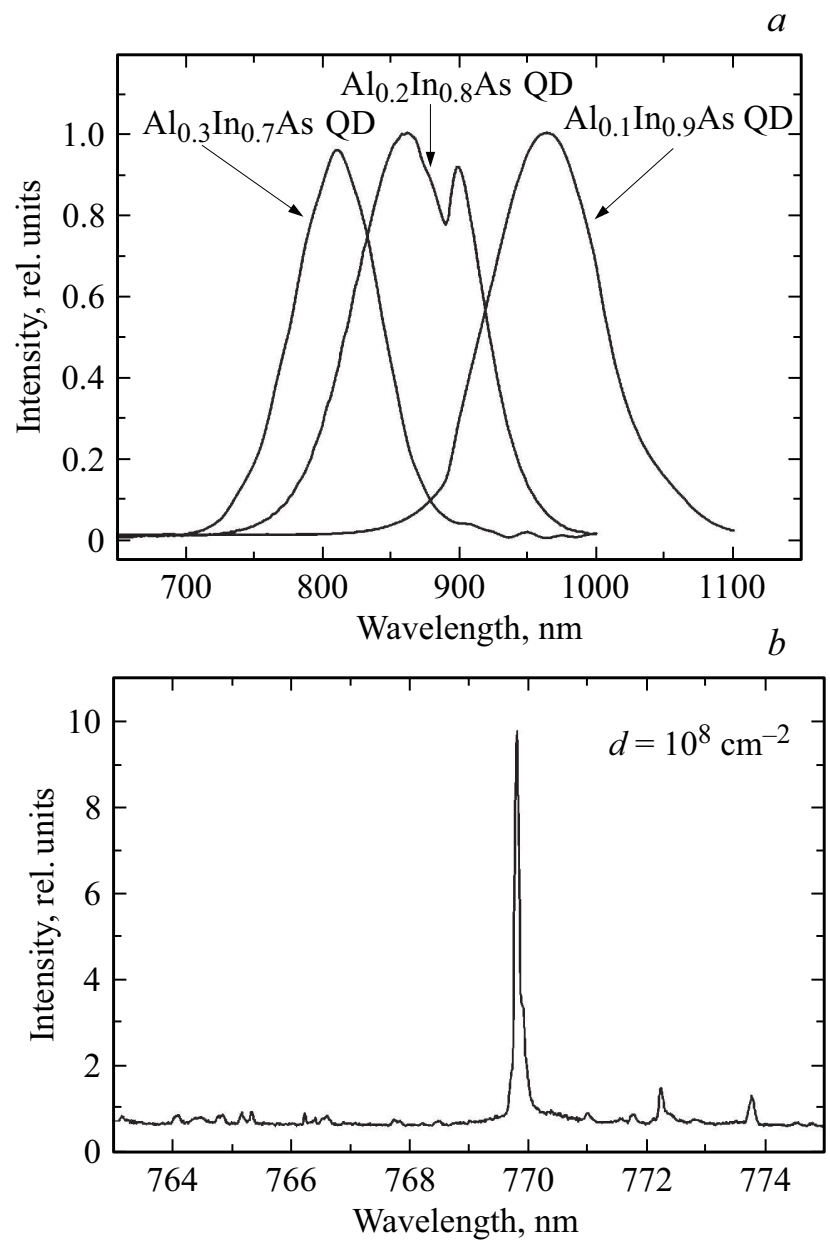

Рис. 1. Спектры макрофотолюминесценции трех структур, содержащих $\mathrm{Al}_{x} \mathrm{In}_{1-x} \mathrm{As}_{2} \mathrm{Al}_{y} \mathrm{Ga}_{1-y} \mathrm{As} \quad$ КТ при $T=295 \mathrm{~K} \quad(a)$. Спектр микролюминесценции структуры $\mathrm{Al}_{0.1} \mathrm{In}_{0.9} \mathrm{As} / \mathrm{Al}_{0.24} \mathrm{Ga}_{0.76} \mathrm{As}$ с плотностью КТ $d \approx 10^{8} \mathrm{~cm}^{-2}$ при $T=10 \mathrm{~K}$, содержащий пик, отвечающий рекомбинации экситона $X$ одиночной КТ $(b)$.

диапазона излучения КТ в коротковолновую область (до 200 нм), включая участок длин волн вблизи 770 нм, представляющий интерес для разработки атмосферных систем квантовой криптографии. Данные, приведенные на рис. 1, $a$, были получены на участках исследуемых структур, содержащих массивы $\mathrm{Al}_{x} \operatorname{In}_{1-x} \mathrm{As}_{2} / \mathrm{Al}_{y} \mathrm{Ga}_{1-y} \mathrm{As}$ КТ высокой плотности $\left(d \geq 10^{10} \mathrm{~cm}^{-2}\right)$. Для исследования характеристик одиночных квантовых точек требуются области с низкой плотностью $d \leq 10^{8} \mathrm{~cm}^{-2}$. Для решения этой задачи был использован следующий подход, рост слоя $\mathrm{Al}_{x} \mathrm{In}_{1-x} \mathrm{As}$ осуществлялся без вращения подложки. Это задавало градиент толщины данного слоя, в результате чего структура содержала участки КТ различной плотности в диапазоне от нулевой до предельно высокой $d \approx 10^{11} \mathrm{~cm}^{-2}$. Наличие областей с низкой плотностью КТ на синтезированных структурах позволило уверенно адресоваться к одиночным КТ и исследовать их оптические характеристики с использованием методики микрофотолюминесценции. На рис. $1, b$ приведен спектр структуры $\mathrm{Al}_{0.1} \mathrm{In}_{0.9} \mathrm{As} / \mathrm{Al}_{0.24} \mathrm{Ga}_{0.76} \mathrm{As} \quad \mathrm{KT}$, полученный на участке с плотностью $d \approx 10^{8} \mathrm{~cm}^{-2}$ при $T=10 \mathrm{~K}$. Спектр содержит узкий пик, отвечающий рекомбинации экситона $X$ одиночной $\mathrm{Al}_{0.1} \mathrm{In}_{0.9} \mathrm{As} / \mathrm{Al}_{0.24} \mathrm{Ga}_{0.76} \mathrm{As}-$ квантовой точки.

Статистика излучения одиночных КТ анализировалась на основе измерения парных фотонных корреляций, получаемых с помощью интерферометра ХБТ. Измерялся коррелятор 2-го порядка, или коррелятор интенсивностей $I$ :

$$
g^{2}(\tau)=\frac{\left\langle I_{1}(t) I_{2}(t+\tau)\right\rangle}{\left\langle I_{1}(t)\right\rangle\left\langle I_{2}(t)\right\rangle} .
$$

В (1) угловые скобки означают усреднение по ансамблю, $\tau$ - время задержки в регистрируемой паре фотонов, задаваемое электронным блоком интерферометра ХБТ. При пуассоновской статистике излучения $g^{2}(\tau)=1$, что означает полное отсутствие корреляции излучения фотонов [20,21]. Для суперпуассоновского излучения, где проявляется эффект группировки (bunching) фотонов: $1<g^{2}(\tau)<2$. При субпуассоновской статистике излучения (разгуппировка фотонов (antibunching)): $g^{2}(\tau)<1$. В идеальном случае, когда рассматриваются однофотонные фоковские состояния и задержка регистрации пар фотонов $\tau=0$, интерферометр ХБТ не зарегистрирует совпадений: $g^{2}(0)=0$.

На рис. 2 представлена зависимость $g^{2}(\tau)$, измеренная для экситонного пика одиночной $\mathrm{Al}_{0.1} \mathrm{In}_{0.9} \mathrm{As} /$ $\mathrm{Al}_{0.24} \mathrm{Ga}_{0.76} \mathrm{As}$ КТ (рис. 1,b). При $\tau=0$ зависимость $g^{2}(\tau)$ имеет ярко выраженный минимум, $g^{2}(0) \approx 0.46$, что указывает на субпуассоновский тип статистики излучения. Это является прямым подтверждением возможности создания излучателей одиночных фотонов на базе $\mathrm{Al}_{0.1} \operatorname{In}_{0.9}$ As KT.

Расщепление экситонных состояний $\Delta E_{F S}$ было измерено для 30 одиночных $\mathrm{Al}_{0.1} \mathrm{In}_{0.9} \mathrm{As} / \mathrm{Al}_{0.24} \mathrm{Ga}_{0.76} \mathrm{As}$ КТ. Полученные экспериментальные данные представлены на рис. 3. Из рисунка видно, что в ряде КТ расщепление экситонных состояний значительно, и

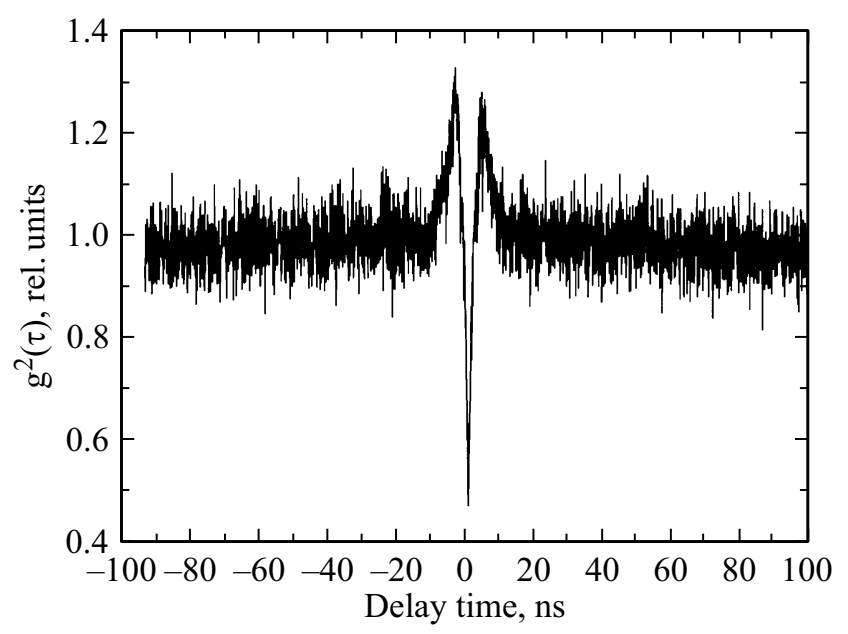

Рис. 2. Зависимость $g^{2}(\tau)$, демонстрирующая субпуассоновский тип статистики излучения $\mathrm{Al}_{0.1} \mathrm{In}_{0.9} \mathrm{As} / \mathrm{Al}_{0.24} \mathrm{Ga}_{0.76} \mathrm{As}$ KT. 


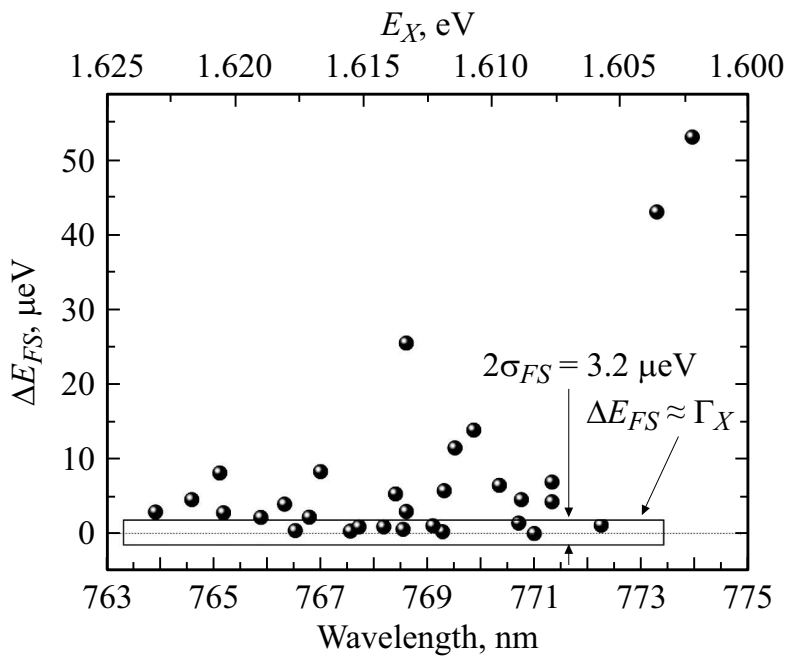

Рис. 3. Расщепление экситонных состояний $\Delta E_{F S}$ для 30 $\mathrm{Al}_{0.1} \mathrm{In}_{0.9} \mathrm{As} / \mathrm{Al}_{0.24} \mathrm{Ga}_{0.76} \mathrm{As}$ KT. В прямоугольной рамке выделены экспериментальные точки, для которых $\Delta E_{F S}$ сравнимо с естественной шириной экситонных состояний.
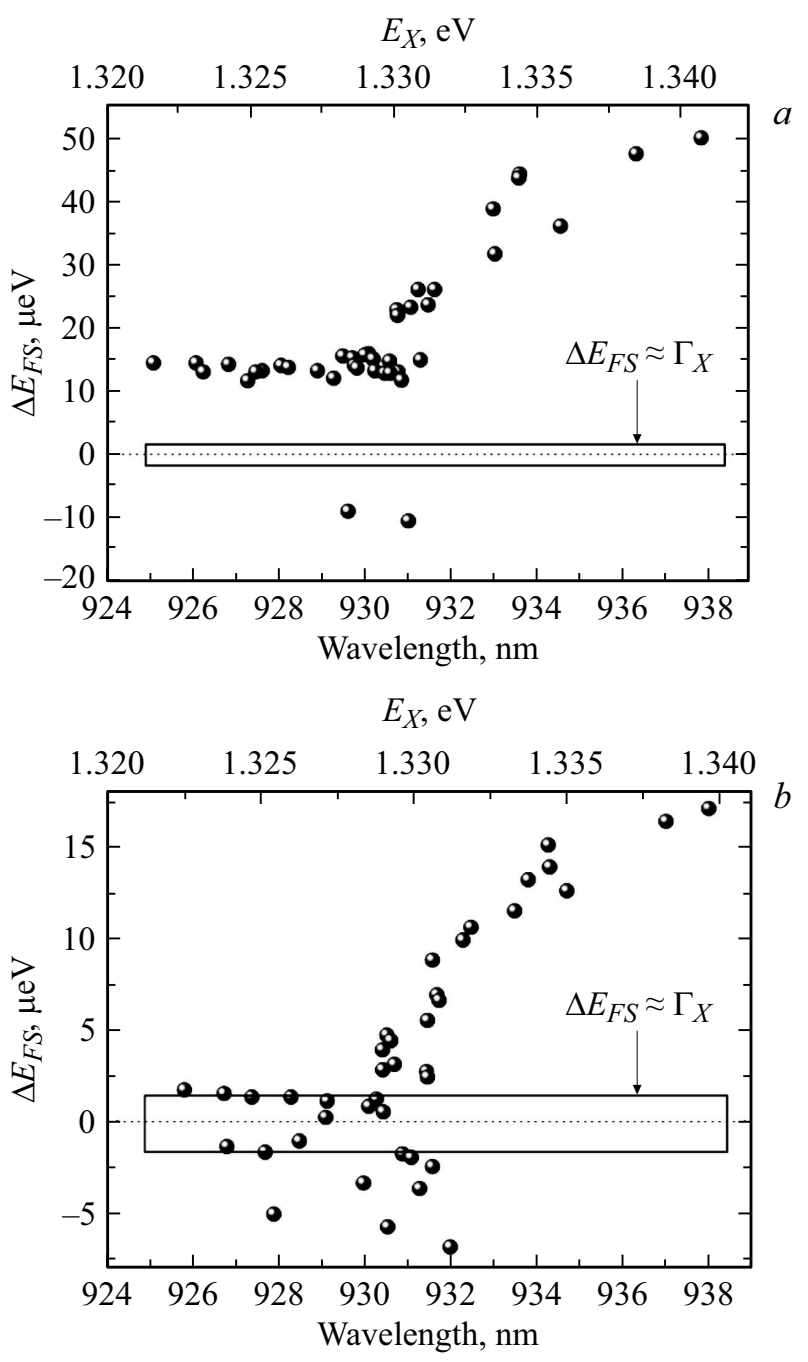

Рис. 4. Расщепление экситонных состояний $\Delta E_{F S}$ для 40 (111)InGaAs KT. $a-$ условия роста структуры $T_{\text {sub }}=480^{\circ} \mathrm{C}$, $t_{Q D}=33 \mathrm{c}, \quad \tau_{\mathrm{GI}}=60 \mathrm{c} ; \quad b-$ условия роста структуры $T_{\text {sub }}=480^{\circ} \mathrm{C}, t_{Q D}=30 \mathrm{c}, \tau_{\mathrm{GI}}=0 \mathrm{c}$. максимальная зарегистрированная величина составляет $\Delta E_{F S} \approx 50$ мкэВ. При этом большая часть КТ характеризуется $\Delta E_{F S} \leq 10$ мкэВ. И наконец, примерно для $30 \%$ исследованных $\mathrm{Al}_{0.1} \mathrm{In}_{0.9} \mathrm{As} / \mathrm{Al}_{0.24} \mathrm{Ga}_{0.76} \mathrm{As}$ KТ выполняется условие $\Delta E_{F S} \approx \Gamma=\hbar / \tau_{X}$. На рис. 3 эта группа КТ отмечена прямоугольной рамкой. Очевидно, что данная выборка КТ представляет большой интерес для создания ИФП на основе $\mathrm{Al}_{x} \mathrm{In}_{1-x} \mathrm{As}$ квантовых точек.

При исследовании расщепления экситонных состояний системы (111)InGaAs КТ было установлено, что параметр $\Delta E_{F S}$ может варьироваться в широких пределах от образца к образцу в зависимости от режимов его выращивания. Для каждой из выращенных структур определялись параметры $\Delta E_{F S}$ для 40 одиночных КТ. В ряде структур величина $\Delta E_{F S}$ могла достигать десятков мкэВ, при этом КТ, для которых выполняется условие $\Delta E_{F S} \approx \Gamma$, выявлены не были. Пример такой структуры приведен на рис. 4, $a$. Структура выращивалась при условиях $T_{\mathrm{sub}}=480^{\circ} \mathrm{C}, t_{Q D}=33 \mathrm{c}\left(h_{\mathrm{In}} \approx 2.2 \mathrm{MC}\right)$ и время ростовой паузы $\tau_{\mathrm{GI}}=60 \mathrm{c}$. Прямоугольной рамкой на рис. $4, a$ отмечено условие $\Delta E_{F S} \approx \Gamma$.

Оптимальными режимами роста для получения КТ c $\Delta E_{F S} \approx \Gamma$ были признаны следующие: $T_{\text {sub }}=480^{\circ}$, $t=30 \mathrm{c}\left(h_{\mathrm{In}} \approx 2.0 \mathrm{MC}\right)$ и нулевое время ростовой паузы $\tau_{\mathrm{GI}}=0 \mathrm{c}$. Результаты по исследованию $\Delta E_{F S}$ такой структуры приведены на рис. $4, b$. Для большинства КТ $\Delta E_{F S}$ не превышает 15 мкэВ. Примерно для $30 \%$ исследованных КТ в этой структуре выполняется условие $\Delta E_{F S} \approx \Gamma=\hbar / \tau_{X}$. На рис. $4, b$ эта группа КТ выделена рамкой.

\section{4. Заключение}

Таким образом, в данной работе исследована система квантовых точек на основе AlInAs и (111)InGaAs. Использование широкозонных твердых растворов $\mathrm{Al}_{x} \mathrm{In}_{1-x} \mathrm{As}$ в качестве основы КТ позволило существенно расширить спектральный диапазон излучения в коротковолновую область, включая участок длин волн вблизи 770 нм, представляющий интерес для разработки аэрокосмических систем квантовой криптографии. На участке длин волн вблизи 770 нм исследована тонкая структура экситонных состояний квантовых точек и показано, что для набора КТ величина расщепления экситонных состояний сравнима с естественной шириной экситонных линий. При исследовании расщепления экситонных состояний системы (111)InGaAs KT было установлено, что параметр $\Delta E_{F S}$ может варьироваться в широких пределах от образца к образцу в зависимости от режимов его выращивания. Были определены оптимальные режимы роста для получения КТ с $\Delta E_{F S} \approx \Gamma$. При использовании данных режимов для $\sim 30 \%$ КТ выполняется условие $\Delta E_{F S} \approx \Gamma$. Полученные данные представляют интерес для создания излучателей фотонных пар на основе AlInAs- и (111)InGaAs-квантовых точек.

Работа выполнена при частичной поддержке РФФИ, грант № 16-52-12023. 


\section{Список литературы}

[1] N. Gisin, G. Ribordy, W. Tittel, H. Zbinden. Rev. Mod. Phys., 74 (1), 145 (2002).

[2] В.А. Гайслер, А.В. Гайслер, А.С. Ярошевич, И.А. Деребезов, М.М. Качанова, Ю.А. Живодков, Т.А. Гаврилова, А.С. Медведев, Л.А. Ненашева, К.В. Грачев, В.К. Сандырев, А.С. Кожухов, В.М. Шаяхметов, А.К. Калагин, А.К. Бакаров, Д.В. Дмитриев, А.И. Торопов, Д.В. Щеглов, А.В. Латышев, А.Л. Асеев. ФТП, 49 (1), 35 (2015).

[3] A. Lochmann, E. Stock, O. Schulz, J.A. Tofflinger, W. Unrau, A. Toropov, A. Bakarov, V. Haisler, D. Bimberg. Electron. Lett., 45 (13), 566 (2009).

[4] O. Benson, C. Santori, M. Pelton, Y. Yamamoto. Phys. Rev. Lett., 84, 2513 (2000).

[5] D. Bimberg, E. Stock, A. Lochmann, A. Schliva, J.A. Töfflinger, W. Unrau, M. Münnix, S. Rodt, V.A. Haisler, A.I. Toropov, A. Bakarov. IEEE Photon. J., 1 (1), 58 (2009).

[6] А.В. Гайслер, И.А. Деребезов, А.С. Ярошевич, А.К. Калагин, А.К. Бакаров, А.И. Торопов, Д.В. Щеглов, В.А. Гайслер, А.В. Латышев, А.Л. Асеев. Письма ЖЭТФ, 97, 313 (2013).

[7] R. Seguin, A. Schliwa, S. Rodt, K. Pötschke, U.W. Pohl, D. Bimberg. Phys. Rev. Lett., 95, 257402 (2005).

[8] R. Seguin, A. Schliwa, S. Rodt, K. Pötschke, U.W. Pohl, D. Bimberg. Physica E, 32, 101 (2006).

[9] M. Povolotskyi, A. Di Carlo, S. Birner. Phys. Status Solidi C, 1 (6), 1511 (2004).

[10] R. Singh, G. Bester. Phys. Rev. Lett., 103, 063601 (2009).

[11] A. Schliwa, M. Winkelnkemper, A. Lochmann, E. Stock, D. Bimberg. Phys. Rev. B, 80, 161307 (2009).

[12] A. Mohan, M. Felici, P. Gallo, B. Dwir, A. Rudra, J. Faist, E. Kapon. Nature Photonics, 4, 302 (2010).

[13] N. Gisin, G. Ribordy, W. Tittel, H. Zbinden. Rev. Mod. Phys., 74, 145 (2002).

[14] L.H. Li, N. Chauvin, G. Patriarche, B. Alloing, A. Fiore. J. Appl. Phys., 104, 08358 (2008).

[15] T.J. Krzyzewski, T.S. Jones. J. Appl. Phys., 96, 668 (2004).

[16] L. Muller-Kirsch, R. Heitz, U.W. Pohl, D. Bimberg. Appl. Phys. Lett., 79, 1027 (2001).

[17] U.W. Pohl, K. Potschke, A. Schliwa, F. Guffarth, D. Bimberg, N.D. Zakharov, P. Werner, M.B. Lifshits, V.A. Shchukin, D.E. Jesson. Phys. Rev. B, 72, 245332 (2005).

[18] S. Panyakeow. Eng. J., 13 (1), 51 (2009).

[19] Z. Gong, Z.C. Niu, S.S. Huang, Z.D. Fang, B.Q. Sun, J.B. Xia. Appl. Phys. Lett., 87, 093116 (2005).

[20] Single Quantum Dots, Fundamentals, Applications and New Concepts, ed. by P. Michler (Berlin, Springer Verlag, 2003).

[21] Single Semiconductor Quantum Dots, ed. by P. Michler (Berlin, Springer Verlag, 2009).

\section{Spectroscopy of single AllnAs and (111)InGaAs quantum dots}

\author{
I.A. Derebezov 1,2, V.A. Gaisler ${ }^{1}$, A.V. Gaisler ${ }^{1}$, \\ D.V. Dmitriev ${ }^{1}$, A.I. Toropov ${ }^{1}$, M. von Helversen ${ }^{3}$, \\ C. de la Haye ${ }^{3}$, S. Bounouar ${ }^{3}$, S. Reitzenstein ${ }^{3}$ \\ ${ }^{1}$ Institute of Semiconductor Physics, \\ Siberian Branch of Russian Academy of Sciences, \\ 630090 Novosibirsk, Russia \\ ${ }^{2}$ Siberian State University of Telecommunications \\ and Information Science, \\ 630102 Novosibirsk, Russia \\ ${ }^{3}$ Technische Universität Berlin, Institut fü \\ Festkörperphysik Eugene-Wigner-Gebäude, \\ D-10623 Berlin, Federal Republic of Germany
}

Abstract A systems of quantum dots on the basis of AlInAs and (111)InGaAs have been studied. The usage of broadband $\mathrm{Al}_{x} \mathrm{In}_{1-x}$ As solid solutions as the basis of quantum dots makes it possible to expand considerably the spectral emission range into the short-wave region, including the wavelength region near $770 \mathrm{~nm}$ being of interest for the design of aerospace systems of quantum cryptography. The fine structure of exciton states of AlInAs and (111)InGaAs quantum dots is studied. It is shown that the splitting of exciton states is comparable with the natural width of exciton lines, which is of great interest for the design of emitters of pairs of entangled photons on the basis of these quantum dots.

Редактор Г.А. Оганесян 